\title{
Confirmation and fine-mapping of a major QTL for resistance to infectious pancreatic necrosis in Atlantic salmon (Salmo salar): population-level associations between markers and trait Thomas Moen*1,2,3, Matthew Baranski2,3, Anna K Sonesson ${ }^{2}$ and Sissel Kjøglum ${ }^{1}$
}

Address: ${ }^{1}$ Aqua Gen AS, Trondheim, Norway, ${ }^{2}$ Nofima Marine, Ås, Norway and ${ }^{3}$ CIGENE - Centre of Integrative Genetics, University of Life Sciences, Ås, Norway

Email: Thomas Moen* - thomas.moen@aquagen.no; Matthew Baranski - matthew.baranski@nofima.no;

Anna K Sonesson - anne.sonesson@nofima.no; Sissel Kjøglum - sissel.kjoglum@aquagen.no

* Corresponding author

Published: 7 August 2009

BMC Genomics 2009, 10:368 doi:10.1186/147|-2164-10-368

This article is available from: http://www.biomedcentral.com/147/-2/64/10/368

(c) 2009 Moen et al; licensee BioMed Central Ltd.

This is an Open Access article distributed under the terms of the Creative Commons Attribution License (http://creativecommons.org/licenses/by/2.0), which permits unrestricted use, distribution, and reproduction in any medium, provided the original work is properly cited.

\begin{abstract}
Background: Infectious pancreatic necrosis (IPN) is one of the most prevalent and economically devastating diseases in Atlantic salmon (Salmo salar) farming worldwide. The disease causes large mortalities at both the fry- and post-smolt stages. Family selection for increased IPN resistance is performed through the use of controlled challenge tests, where survival rates of sib-groups are recorded. However, since challenge-tested animals cannot be used as breeding candidates, withinfamily selection is not performed and only half of the genetic variation for IPN resistance is being exploited. DNA markers linked to quantitative trait loci (QTL) affecting IPN resistance would therefore be a powerful selection tool. The aim of this study was to identify and fine-map QTL for IPN-resistance in Atlantic salmon, for use in marker-assisted selection to increase the rate of genetic improvement for this trait.
\end{abstract}

Results: A genome scan was carried out using 10 large full-sib families of challenge-tested Atlantic salmon post-smolts and microsatellite markers distributed across the genome. One major QTL for IPN-resistance was detected, explaining $29 \%$ and $83 \%$ of the phenotypic and genetic variances, respectively. This QTL mapped to the same location as a QTL recently detected in a Scottish Atlantic salmon population. The QTL was found to be segregating in 10 out of 20 mapping parents, and subsequent fine-mapping with additional markers narrowed the QTL peak to a $4 \mathrm{cM}$ region on linkage group 21. Challenge-tested fry were used to show that the QTL had the same effect on fry as on post-smolt, with the confidence interval for QTL position in fry overlapping the confidence interval found in post-smolts. A total of 178 parents were tested for segregation of the QTL, identifying 72 QTL-heterozygous parents. Genotypes at QTL-heterozygous parents were used to determine linkage phases between alleles at the underlying DNA polymorphism and alleles at single markers or multi-marker haplotypes. One four-marker haplotype was found to be the best predictor of QTL alleles, and was successfully used to deduce genotypes of the underlying polymorphism in $72 \%$ of the parents of the next generation within a breeding nucleus. A highly significant population-level correlation was found between deduced alleles at the underlying polymorphism and survival of offspring groups in the fry challenge test, parents with the three deduced genotypes $(Q Q, Q q, q q)$ having mean offspring mortality rates of $0.13,0.32$, and 0.49 , 
respectively. The frequency of the high-resistance allele $(Q)$ in the population was estimated to be 0.30. Apart from this major QTL, one other experiment-wise significant QTL for IPN-resistance was detected, located on linkage group 4.

Conclusion: The QTL confirmed in this study represents a case of a major gene explaining the bulk of genetic variation for a presumed complex trait. QTL genotypes were deduced within most parents of the 2005 generation of a major breeding company, providing a solid framework for linkage-based MAS within the whole population in subsequent generations. Since haplotype-trait associations valid at the population level were found, there is also a potential for MAS based on linkage disequilibrium (LD). However, in order to use MAS across many generations without reassessment of linkage phases between markers and the underlying polymorphism, the QTL needs to be positioned with even greater accuracy. This will require higher marker densities than are currently available.

\section{Background}

Genomics is beginning to make an impact on animal breeding, by providing DNA markers linked to genes affecting phenotypic traits. Such markers can be used in marker-assisted selection (MAS), selection based partly or fully on DNA marker genotypes. Among the traits relevant for MAS, disease resistance traits are of particular interest, since the phenotypic measurement of these traits is often difficult or expensive and may cause animal suffering.

In aquaculture, disease resistance traits are of particular importance. In intensive culture systems, opportunities for avoidance or escape are minimal. Furthermore, interactions between fish and microbial pathogens that may be harmless under natural conditions often result in disease problems in aquaculture systems because of the added stress from biological, physical and chemical factors [1]. In contrast to farm animals, the animal strains used in aquaculture are usually very recent derivatives of wild strains [2], and therefore have had little time to adapt to the new disease pressures.

The Atlantic salmon (Salmo salar) is, as its relative the rainbow trout (Oncorhyncus mykiss), an important species in modern aquaculture. In the wild, this carnivorous species spends its first 2-5 years in fresh water, but migrates to the sea following the salt-water-acclimatising process known as smoltification. The animal spends 1 to 4 years in the ocean, and then returns to its home river to spawn, burying the eggs in the gravel substrate. The recently hatched fish, termed alevins, live of their yolk sack before they enter the fry stage and emerge from the substrate. The fresh/salt-water (anadromous) life-style of the fish is reflected in aquaculture production, where the fish spend their first $1 / 2$ to 1 year in freshwater tanks and 2 to 3 years in sea cages until they reach market size.

Pathogens are a major problem in salmonid farming as in other areas of aquaculture. In European production of Atlantic salmon, the viral disease infectious pancreatic necrosis (IPN), caused by a double-stranded RNA virus, has for some time ranked among the diseases causing the largest losses [3,4]. In farmed Atlantic salmon, the disease causes mortality at both the freshwater (fry) stage and at the post-smolt stage shortly after transfer to seawater. The disease causes necrosis of pancreatic cells and liver cells, resulting in lethargy and sudden mortality. IPN is an endemic disease that affected $40-70 \%$ of all Norwegian seawater salmon-farming sites during the years 19942004 , and $30-40 \%$ of freshwater hatcheries during the same period [5]. Mortality during outbreaks has been estimated to be $10-20 \%$ on average, varying from zero to almost $100 \%$ at individual sites [5]. Vaccines against IPN have been developed and are being used, but the protection is variable and not complete [6]. In the wild, the virus does not seem to cause mortalities, although wild fish may be carriers of the virus.

The Atlantic salmon has been vital to the application of modern animal breeding to aquaculture. In the early 1970s, a breeding programme was established in Norway, based on fish from different Norwegian rivers [7]. Later, other breeding programmes have been initiated in several countries [8]. Typical traits under selection are growth rate, age at sexual maturation, filet colour and other quality traits, as well as disease resistance traits. Selection for disease resistance may be based on field trials or on controlled challenge tests, but in either case, challenge-tested fish are not allowed as breeding candidates, and the breeding values of the candidates are based solely on survival rates of siblings and other relatives. Criteria for direct selection among candidates, in the form of quantitative trait loci (QTL) for resistance against various diseases, have been sought after for some time in Atlantic salmon and rainbow trout [9-24]. Of particular relevance for IPN in Atlantic salmon was a recent report on the mapping of QTL for resistance against IPN in post-smolts of Scottish origin, based on data from a field trial [25]. In that study, a major QTL was detected that explained 21\% of the phenotypic variation in the data set. The QTL was found to be 
unusually reproducible in the population, segregating in 7 out of 20 mapping parents investigated.

Here, we report on results from a project that has been running partly in parallel with the Scottish study, reproducing the finding of one major QTL by means of a genome scan on salmon of Norwegian origin. We also present the testing of the QTL at the fry stage and the finemapping and further characterisation of the QTL in an extensive genetic material. The results represent, within aquaculture, a rare example of an investigation of a QTL at the level of an entire breeding population, yielding results that are directly applicable for MAS within that population.

\section{Results \\ Phenotypic data}

Ten large full-sib groups of post-smolt from a Norwegian breeding company (Aqua Gen Ltd., Trondheim, Norway) were IPN-challenged-tested by cohabitant challenge in two tanks. The survival curves were found to be very similar across the two tanks, and followed the expected trajectories for an IPN challenge (Figure 1). The overall mortalities in the two tanks were $70.5 \%$ and $77.8 \%$, respectively. Mortality rates of individual families ranged from $51.7 \%$ to $98.5 \%$ (Figure 1 ).

\section{Genome scan}

A genome scan was performed using the 92 first to die and 92 of the survivors (or last to die) within each full-sib family, as well as genotypes on 136 microsatellite markers spanning most of the Atlantic salmon genome (Additional file 1). The information content was relatively high

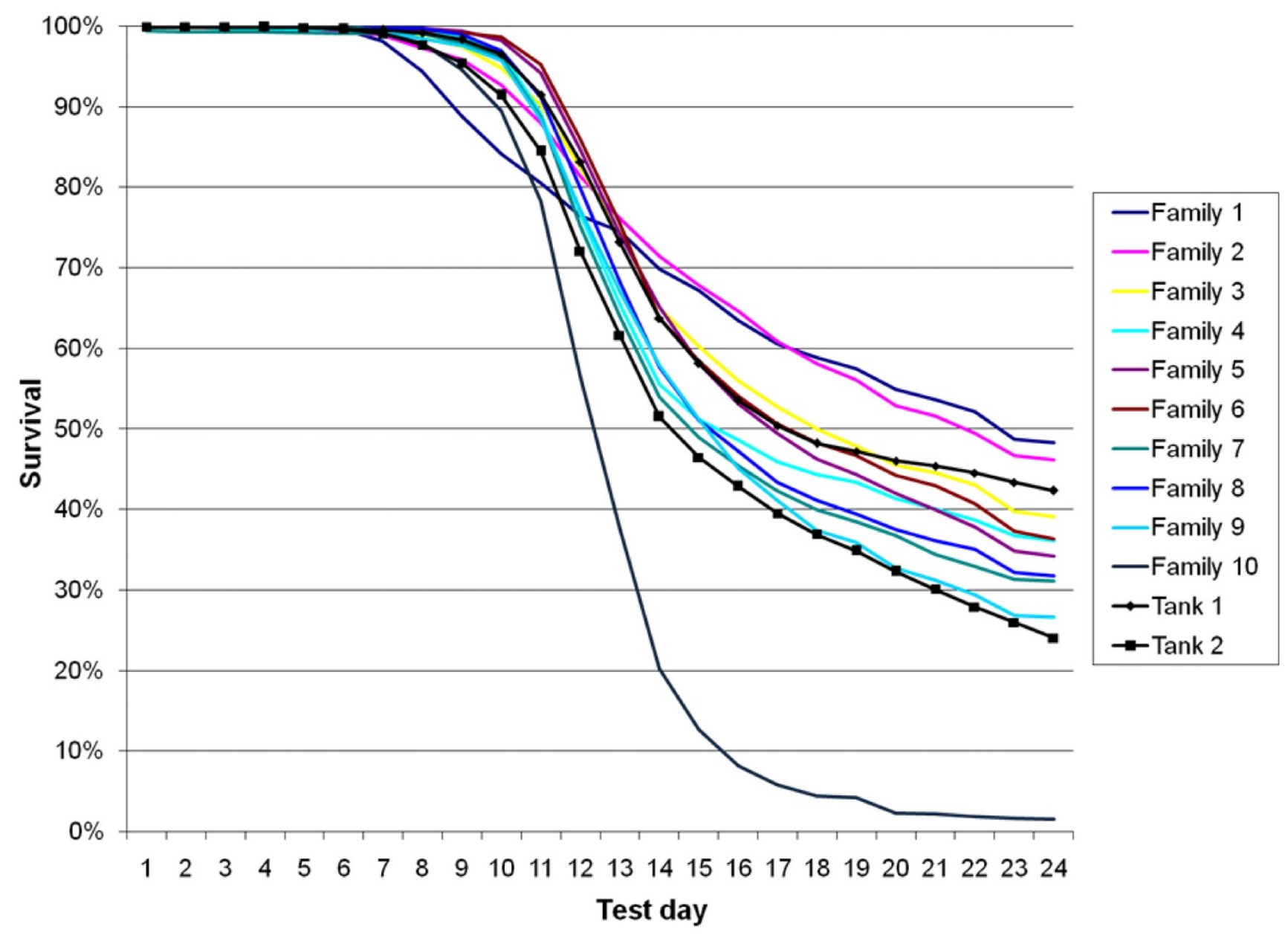

Figure I

Mortality curves from post-smolt challenge test. 
in most regions (Additional file 2). Two genome-wide significant QTL for IPN resistance were identified, one having a major effect and one having a more minor effect. In addition, several suggestive QTL were identified (Table 1, Additional file 2). The major QTL, located on linkage group 21, was responsible for $75.8 \%$ of the phenotypic variation in the data set, translating into $24.6 \%$ of the phenotypic variation after selective genotyping had been taken into account, and consequently $70.3 \%$ of the genetic variation. The one other genome-wide significant QTL was located on linkage group 4, and was responsible for $0.9 \%$ of the genetic variation after selective genotyping had been taken into account.

\section{Linkage-based fine-mapping of major QTL using post- smolts}

To more precisely define the position of the major QTL on linkage group 21, 18 additional microsatellite markers were genotyped in the post-smolt individuals (Table 2). The most likely QTL position was found to be at $25 \mathrm{cM}$, with the 95\% confidence interval for position being at 23-26 cM (Figure 2). The QTL now explained 29\% of the phenotypic variation after correcting for selective genotyping, translating into $83 \%$ of the genetic variation. Out of the 20 mapping parents, 10 were found to be QTL heterozygous $(\mathrm{P}<0.05)$. Out of the 10 QTL heterozygous parents, seven were notably more significant (P-values ranging from $5 \times 10^{-9}$ to $\left.1 \times 10^{-36}\right)$ than the three others (P-values ranging from 0.01 to 0.04 ).

\section{Test of QTL-effect in fry}

Challenge-tested fry, full-sibs of the post-smolts used for the genome scan, were genotyped to investigate whether the QTL had the same effect at the fry stage as at the postsmolt stage, the disease causing mortalities at these two quite different life stages. Chi-square tests performed on groups of fry corresponded well with chi-square tests performed on their post-smolt siblings (Pearson's correlation coefficient $=0.72, \mathrm{P}<0.001$ ). Of the 10 mapping parents found to be QTL heterozygous based on post-smolt data, seven were identified as QTL heterozygous in fry; these were the seven being most significant for the QTL in postsmolts. Vice versa, one parent was found to be QTL heterozygous based on fry data but not on post-smolt data, although it was close to being declared QTL heterozygous in the post-smolt test as well $(P=0.051)$.

\section{Linkage-based fine-mapping of major QTL using fry}

The results had indicated that the major QTL had similar effects on fry as on post-smolts, meaning that genetic material from the fry challenge tests, in which all families in the Aqua Gen breeding nucleus had been tested, could be used to fine map and further characterise the QTL. Consequently, 108 additional full-sib groups of challenge-tested fry, representing 178 parents, were genotyped using markers located on linkage group 21. The position of the QTL based on fry data corresponded well with the position based on post-smolt data, the 95\% confidence interval for position being at 19-26 cM (Figure 2). The QTL explained $16.9 \%$ of the phenotypic variation in the data set following correction for selective genotyping, translating into $48.4 \%$ of the genetic variation. Out of 178

Table I: Significant and suggestive QTL for resistance to IPN

\begin{tabular}{|c|c|c|c|c|c|}
\hline Linkage group & Position (cM) & $\mathrm{F}$ & No of informative parents & Chromosome-wise p-value & Proportion of phenotypic variance \\
\hline 21 & 6 & $43.34 \dagger$ & 19 & $\approx 0 \dagger$ & $24.6 \%$ \\
\hline 4 & 9 & $2.44 *$ & 20 & 0.001 & $0.9 \%$ \\
\hline 13 & 18 & 2.37 & 18 & 0.003 & $0.9 \%$ \\
\hline 22 & 65 & 2.31 & 19 & 0.007 & $0.9 \%$ \\
\hline 10 & 27 & 2.26 & 19 & 0.008 & $0.8 \%$ \\
\hline $17 \mathrm{a}$ & 1 & 2.25 & 18 & 0.004 & $0.8 \%$ \\
\hline 25 & 7 & 2.21 & 19 & 0.003 & $0.8 \%$ \\
\hline 2 & 49 & 2.08 & 20 & 0.010 & $0.7 \%$ \\
\hline 11 & 44 & 2.04 & 19 & 0.009 & $0.7 \%$ \\
\hline 3 & 31 & 2.02 & 20 & 0.005 & $0.7 \%$ \\
\hline $17 \mathrm{~b}$ & 14 & 2.00 & 17 & 0.018 & $0.6 \%$ \\
\hline 12 & 0 & 1.82 & 20 & 0.049 & $0.5 \%$ \\
\hline 28 & I & 1.79 & 19 & 0.047 & $0.5 \%$ \\
\hline 24 & 0 & 1.78 & 19 & 0.024 & $0.5 \%$ \\
\hline 16 & 0 & 1.61 & 20 & 0.045 & $0.4 \%$ \\
\hline
\end{tabular}

$* P<0.05$ (genome-wide)

$\dagger P \approx 0$ ( $P$-value too low to be determined by permutation testing)

QTL were defined as suggestive if they were chromosome-wise significant at $P<0.05$, while not experiment-wise significant at $P<0.05$. The $F$ ratio has degrees of freedom equal to the number of informative parents and the number of records (offspring) minus two times the number of informative parents [38]; the latter ranging from 3177 to 3737 depending on the number of marker-informative offspring. The proportion of phenotypic variance is the proportion of phenotypic variance explained by the QTL, after correction for selective genotyping. 
Table 2: Microsatellite markers on linkage group $2 \mathrm{I}$ used in this study

\begin{tabular}{lrr}
\hline Marker & Position on female map (cM) & Position on male map (cM) \\
\hline OMMII97 & 0 & 0 \\
Omi27TUFa & 1.2 & $>50$ \\
Rsa354 & 2.7 & $>50$ \\
BX867I5I/iia & 3.4 & $>50$ \\
Omy 1002UW & 6.5 & $>50$ \\
CL68994 & 6.7 & $>50$ \\
SsaDI57a & 8.2 & $>50$ \\
OmyRGT44TUF & 8.9 & $>50$ \\
Ssa0800BSFU & 12.4 & $>50$ \\
BHMS217 & 12.7 & $>50$ \\
Rsa476 & 15.1 & $>50$ \\
CLI8304 & 15.3 & $>50$ \\
Ssa0279BSFU & 16.5 & $>50$ \\
Ssa0285BSFU & 22.7 & $>50$ \\
Alu333 & 26.1 & $>50$ \\
Ssa0374BSFU/ii & 26.5 & $>50$ \\
Ssa0680BSFU & 27.2 & $>50$ \\
CLI0332 & 29.8 & $>50$ \\
Ssa0646BSFU & 52.9 & $>50$ \\
Ssa0562BSFU & 56.4 & \\
Ssa0428BSFU & 57.1 & \\
\hline
\end{tabular}

aMarkers used in the genome scan

mapping parents, 72 were found to be segregating the QTL $(\mathrm{P}<0.05)$. Combined linkage disequilibrium/linkage analysis (LDLA) was performed, but could not add to the precision of the QTL position estimate (data not shown). It should be noted that fry were not scanned for QTL outside of linkage group 21. Thus, QTL located outside this linkage group, having an effect on fry but not on post-smolts, could not be detected.

\section{Test for population-level association between markers and} the underlying polymorphism

Levels of linkage disequilibrium (LD) between markers in the QTL region showed that there was significant LD between closely linked markers on the linkage group as a whole (Figure 3). Within the QTL region, inter-marker $\mathrm{r}^{2}$ values as high as 0.53 were found (Table 3 ). These findings indicated that there could be substantial LD between markers in the QTL region and the polymorphism underlying the QTL. Therefore, markers in the QTL region, as well as haplotypes made from these markers, were tested for their ability to predict alleles at the underlying polymorphism. This analysis was based on the 72 parents found to be QTL heterozygous, since alleles at the underlying polymorphism, and the linkage phases between alleles at the underlying polymorphism and marker alleles, were known for these parents. A four-marker haplotype was found to be the best predictor of alleles at the underlying polymorphism (Table 4); out of 41 alleles found among the 72 QTL heterozygous parents at this haplotype, only three were not exclusively linked to either $Q$ (high-resistance allele) or $q$ (low-resistance allele) (Addi- tional file 3). When linkage phases deduced between $Q / q$ and the four-marker haplotype alleles were extrapolated on the 78 remaining mapping parents with known haplotypes (28 parents had missing marker data), 11 parents were found to be $Q Q, 13$ were $Q q$, and 54 were qq. Thus, a significant homozygote excess (Pearson's $X^{2}$ for deviation from Hardy-Weinberg expectations $=21.18, \mathrm{P}<$ 0.0001 ) was found among the parents that were a priori believed to be QTL homozygous based on linkage analysis (i.e. on the earlier test for QTL based on challenge-tested offspring). Taken together, these results showed that the four-marker haplotype was a good, but not perfect, indicator of allele at the underlying polymorphism. They also gave credit to the one-gene, two-allele model that had implicitly been assumed.

\section{Characterisation of the underlying polymorphism at the population level}

We now were in a position where we could characterise the segregation of the underlying gene at the population level. An additional 214 animals from among the 2005 broodstock (i.e. the parents of the 2005 generation; Figure 4) were therefore genotyped for the four-marker haplotype. Unphased haplotype data from these animals were combined with phased data from the 178 previously investigated mapping parents to deduce haplotypes at the population level, whereupon alleles at the underlying polymorphism were inferred on the basis of haplotypes found in $Q q$ animals (with the three ambiguous haplotypes being classified according to the most prevalent relationship within that haplotype). For 330 out of a total of 


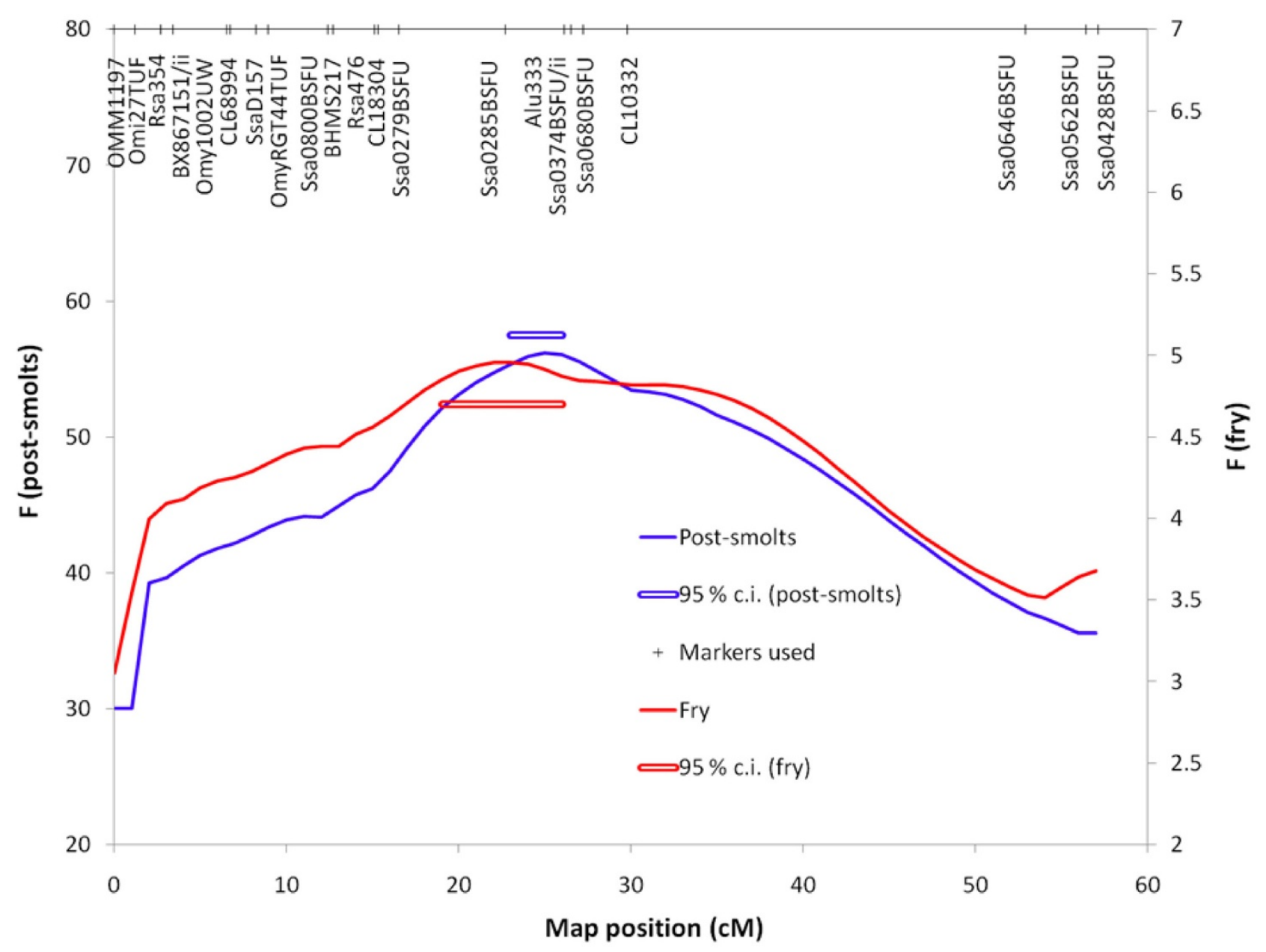

Figure 2

Fine mapping of major IPN-resistance using post-smolt and fry material. The DNA markers used are indicated at the top of the graph. The $95 \%$ bootstrap confidence intervals for position is indicated by horizontal bars.

392 genotyped broodstock animals, both alleles at the underlying polymorphism could be deduced (Figure 4). Of these, 15 were $Q Q, 177$ were $Q q$, and 138 were $q q$, leading to a population level allele frequency estimate for $Q$ of 0.30 . Genotype at the underlying polymorphism had a highly significant $\left(P<10^{-21}\right)$ effect on survival rates of offspring, with the average progeny mortality rates for parents with the $Q Q, Q q$, and $q q$ genotypes being $0.13,0.32$, and 0.49 , respectively (Figure 5). The dominance effect was non-significant, indicating a purely additive mode of inheritance. The genotype of the underlying polymorphism was responsible for $23 \%$ of the trait variance in this test.

\section{Discussion}

The genome scan for QTL affecting IPN resistance confirmed a major QTL explaining nearly all of the genetic variation for this trait. This implies that, in this population, the genetic component of resistance to IPN is largely under the control of one QTL, presumably corresponding to one gene, a surprising find given that disease resistance traits are generally presumed to be complex. The results agree remarkably well with the other study identifying a major QTL for IPN-resistance in Atlantic salmon [25]. In both studies the QTL explained 25\% of the phenotypic variation, it segregated in a large fraction of mapping parents ( 7 out of 20 parents in the Scottish study; 72 out of 176 'fry' mapping parents in the present study), and the estimated QTL positions were highly similar. Only a single marker (Alu333) was shared between the two studies in the vicinity of the QTL, but the position of this marker relative to the QTL peak was very consistent. Thus, it seems reasonable to assume that the two studies have in fact identified one and the same QTL. 


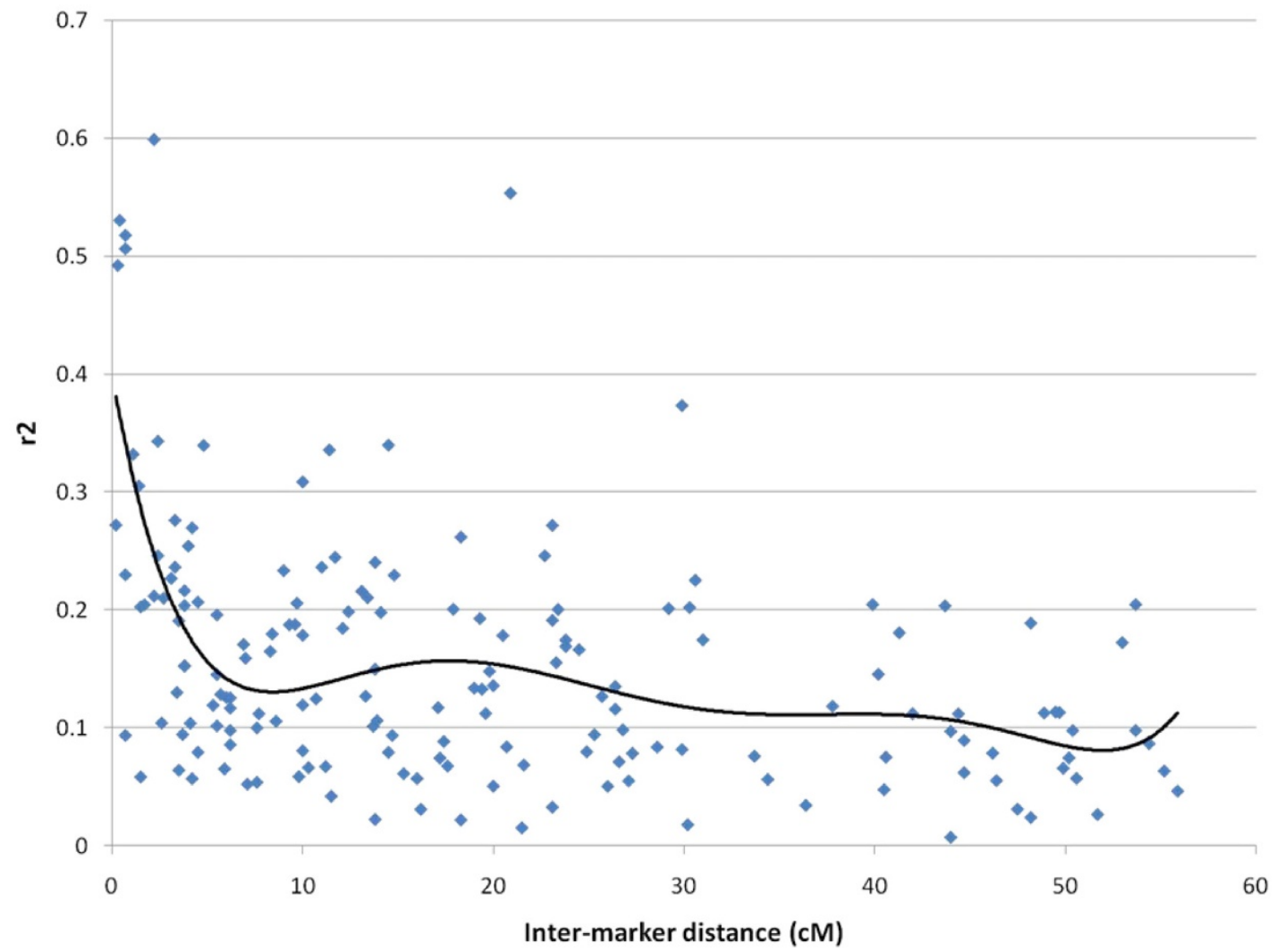

\section{Figure 3}

Levels of inter-marker LD on linkage group 2 I plotted by genetic distance. The measure of LD is the square of the correlation coefficient, $r^{2}$. All possible paired combinations of the 21 genotyped microsatellites were plotted. A $6^{\text {th }}$ degree polynomial was fitted to the graph.

In terms of the minor QTL identified, on the other hand, the overlap between the two studies was less striking. In the Scottish study, one other genome-wide significant QTL was detected, located on linkage group 26, in addition to a chromosome-wide significant QTL in linkage group 19. Of these, only the QTL on linkage group 26 was found to be harbour a (suggestive) QTL in the present work (linkage group 26 in Houston et al. [25] corresponds to linkage group 28 from the present work). The one other genome-significant QTL identified in the present study was identified on linkage group 4 . These differences could be due to differences between the populations studied, with some polymorphisms segregating in one populnation but not in others, or they could be related to sampling since each population was represented by only a limited number of mapping parents in the genome scans. Also, one cannot rule out that some of the QTL could be false positives.

The major QTL on linkage group 21 was found to explain a large fraction of the phenotypic variance in both the post-smolt and fry data sets. In general, the fraction of var-

Table 3: Levels of LD $\left(r^{2}\right)$ between markers in the vicinity of the major QTL for IPN-resistance

\begin{tabular}{|c|c|c|c|c|c|}
\hline & Ssa0279BSFU & Ssa0285BSFU & Alu333 & Ssa0374BSFU/ii & Ssa0680BSFU \\
\hline Ssa0279BSFU & - & 0.13 & 0.19 & 0.31 & 0.12 \\
\hline Ssa0285BSFU & 6.2 & - & 0.13 & 0.15 & 0.08 \\
\hline Alu333 & 9.6 & 3.4 & - & 0.53 & 0.33 \\
\hline Ssa0374BSFU/ii & 10 & 3.8 & 0.4 & - & 0.52 \\
\hline Ssa0680BSFU & 10.7 & 4.5 & I.I & 0.7 & - \\
\hline
\end{tabular}

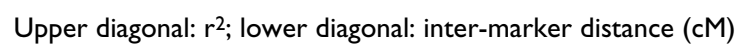


Table 4: Test of the association between marker/haplotype alleles and alleles at the variation underlying the QTL

\begin{tabular}{|c|c|c|c|}
\hline Marker/haplotype & No of alleles & $\mathrm{F}$ & P-value \\
\hline Ssa0279BSFU/ii & 3 & 2.77 & 0.07 \\
\hline Ssa0285BSFU & 6 & 2.94 & 0.01 \\
\hline Alu333 & 6 & 7.96 & I.30E-06 \\
\hline Ssa0374BSFU/ii & 21 & 6.66 & $6.04 \mathrm{E}-12$ \\
\hline Ssa0680BSFU & 5 & 18.60 & $3.01 \mathrm{E}-12$ \\
\hline Ssa0279BSFU-Ssa0285BSFU-Alu333-Ssa0374BSFU/ii & 41 & 25.85 & $4.54 \mathrm{E}-38$ \\
\hline Ssa0279BSFU-Ssa0285BSFU-Alu333-Ssa0374BSFU/ii-Ssa0680BSFU & 47 & 21.99 & I.28E-34 \\
\hline
\end{tabular}

iance explained by a QTL detected in a genome scan tends to be overestimated due to the so-called Beavis effect [2628]. It should be noted, however, that the Beavis effect gets less severe as the experimental power increases and as the observed fraction of variance increases. Thus, our own calculations based on the formulas presented by Xu [28] showed that, for a QTL explaining 28\% of the genetic variation, the bias would be almost negligible even if only 100 offspring had been genotyped. Similarly, given the sample sizes of this study, the upward bias would be insignificant even for QTL of very minor effects. We would argue that the fraction of variance explained by the major QTL on linkage group 21 is more likely to have been underestimated, since the correction for selective genotyp- ing assumes that the $9 \%$ least and $9 \%$ most resistant postsmolts were genotyped, while in reality the set of "most resistant animals" was drawn randomly from the $25 \%$ of animals that survived within each family. The fraction of genetic variance explained by the QTL on the post-smolt stage was calculated on the basis of the heritability estimated in fry, since a good estimate of the heritability at the post-smolt stage was not available. The heritabilities at the two life stages are not necessarily identical.

Body weight has been found to be correlated with immune functions in some studies (e.g. [29,30]), an argument for the use of body weight as a covariate in analysis for disease resistance QTL. In this study, individual fish
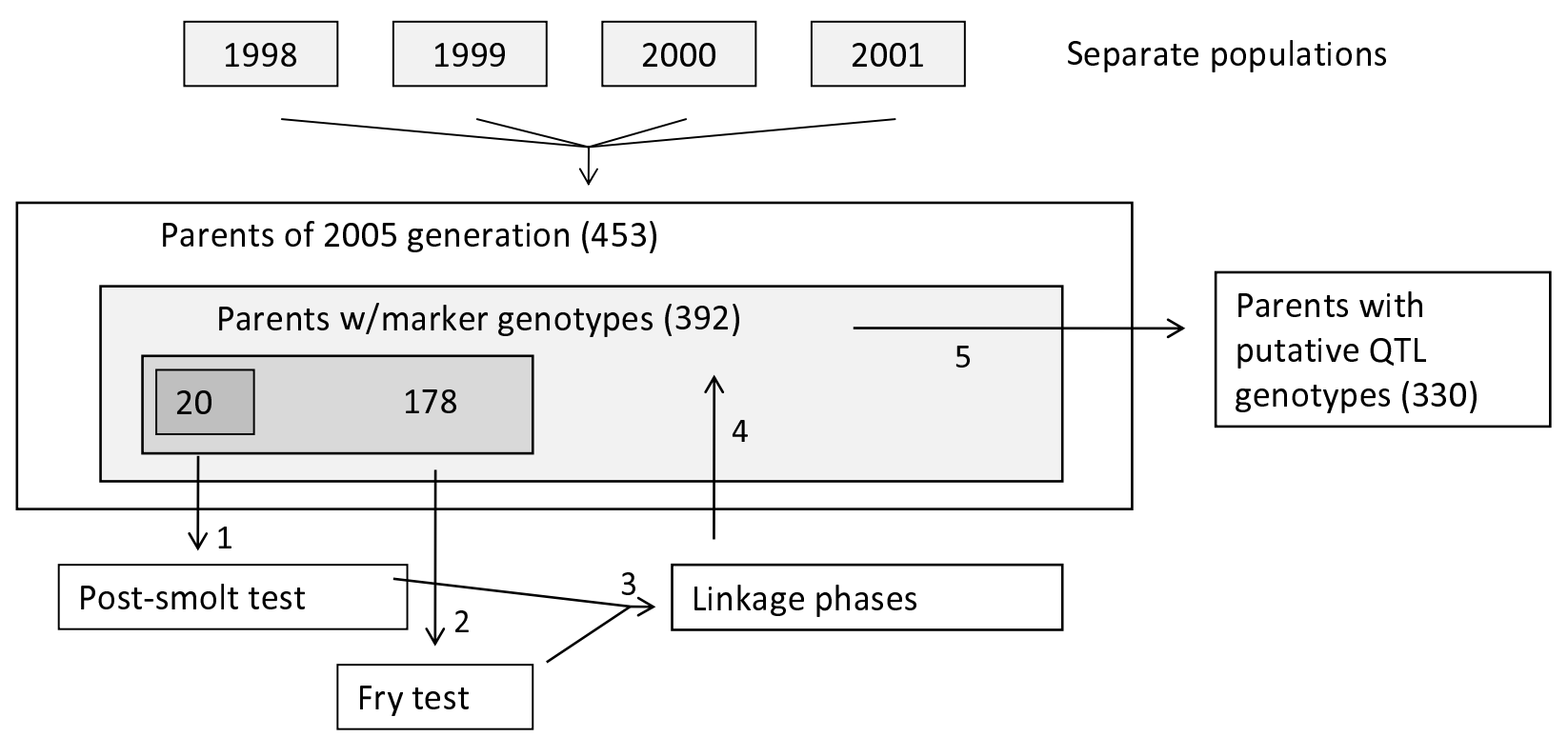

\section{Figure 4}

Animals used in the study. The animals referred to as parents in this study were parents of the 2005 generation of the breeding nucleus of an Atlantic salmon breeding company, and also parents of offspring group that were challenge-tested for IPN at the fry and post-smolt stages. These parents originated from four separate populations (or more specifically, yearclasses of separate populations). Each population (i.e. year class) is denoted according to year of hatching. In the figure, the numbers refer to number of parents, and every box located within another box is a subset of the larger box. The numbers at the arrows indicate the progress of the study. 


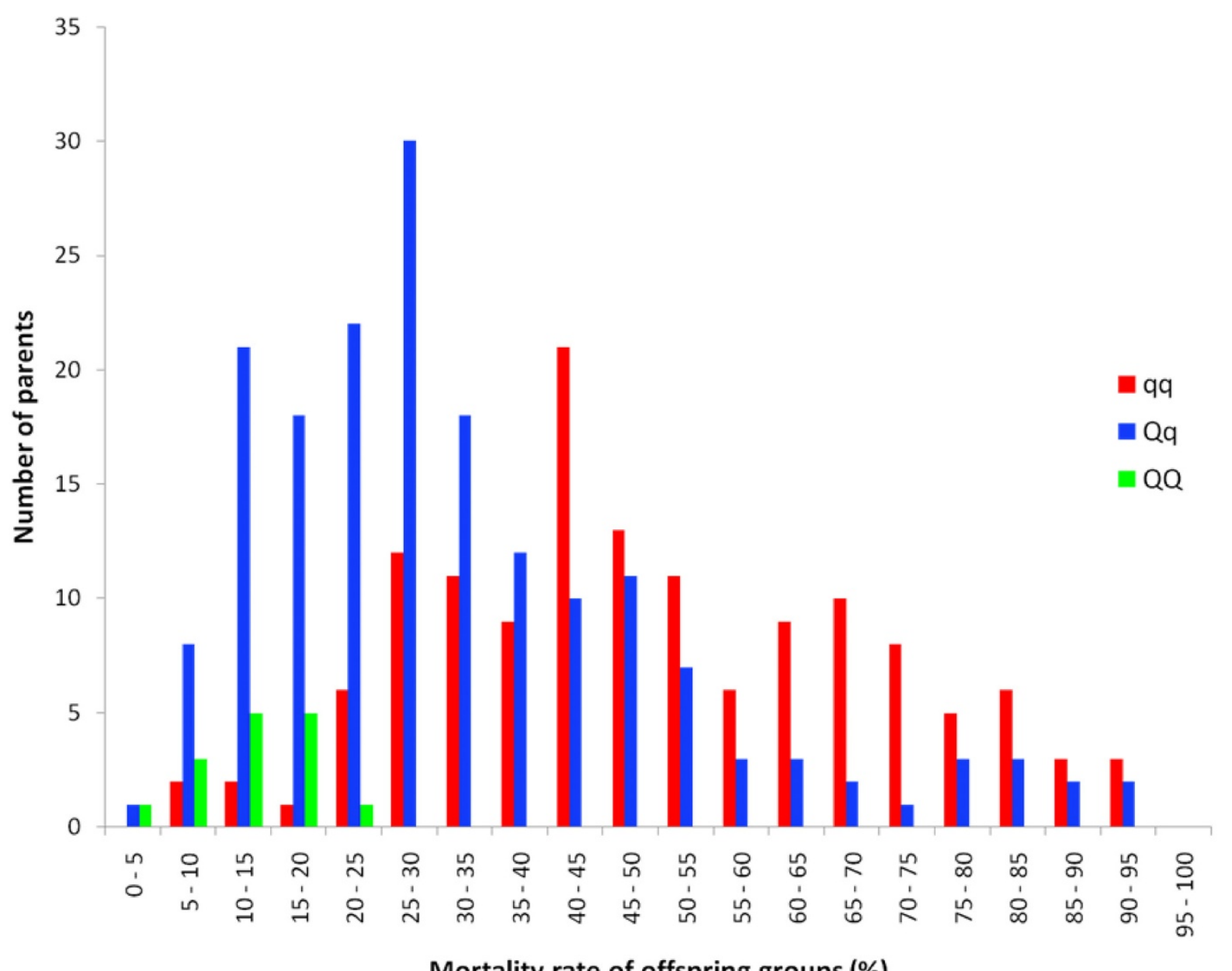

Figure 5

Distribution of challenge test mortality rates among offspring of QQ, Qq, and qq parents. Mortality rates of halfsib groups in the fry challenge test, classified according to the deduced genotype of the common parent at the underlying polymorphism. Each half-sib group contains two full-sib groups, and half-sib mortality rate is the average of the two full-sib mortality rates.

could only be weighed during or after the challenge test, and we were thus not able to include weight as a covariate in the test for QTL (since surviving fish lived longer and thus could have put on extra weight during the challenge). We did, however, analyse the data for body weight QTL, using the 'affected/resistant' status of the fish as a fixed effect in that analysis (Moen et al., in prep.). Linkage group 21 was found not to harbour experiment-wide significant QTL for body weight, meaning that the observed QTL for IPN resistance is not a correlated effect of a QTL for body weight.

For ease of presentation, segregation data from male and female mapping parents were combined in QTL analysis, but only female map distances were used. In large parts of the Atlantic salmon genome, recombination does not occur in males [31], meaning that males do not contribute to the local positioning of QTLs in such regions. In other regions, male recombination rates are very high relative to female recombination rates. The $\mathrm{F}$ curves for linkage group 21 exemplify these particularities well; the sharp increase in the F statistic between OMM1197 and Omi27TUF is caused by these two markers being genetically unlinked in males, even though the female genetic distance between them is only $2 \mathrm{cM}$. For other parts of the linkage group, no male recombination events were found in the whole data set.

The major QTL for IPN-resistance on linkage group 21 had a strong effect in both post-smolts and in fry. These are very distinct life stages, given that fry live in fresh water and post-smolts in salt water. The overlapping confidence 
intervals for QTL position and the concurrence of QTL genotypes and linkage phases between fry and post-smolt stages strongly indicates that the same gene controls IPN resistance at both life stages, although one cannot rule out that two different, but linked genes determine resistance at the two life stages. A functioning adaptive immune system is most probably developed at a later stage than that of the experimental fish in the fry test [32], indicating that the gene underlying the QTL is likely to pertain to the innate immune system. Controlled challenge tests represent somewhat of an 'artificial' environment compared to natural farming conditions, and may lack certain natural stressors that could influence the pattern of resistance to a pathogen. The major QTL on linkage group 21, however, does not appear to be affected by such environmental effects, since it was found to have a large effect both in a field trial [25] and in an experimental trial.

According to inter-marker levels of $\mathrm{r}^{2}$, the extent of linkage disequilibrium increase with decreasing inter-marker distance from $5 \mathrm{cM}$. The increase in LD with decreasing distance is more pronounced in this study than in an earlier study, where the post-smolt mapping families were used to calculate levels of LD across all linkage groups [31]. This is probably due to a higher density of markers being used in the present study relative to the previous study. In spite of significant amounts of LD being present between markers in the QTL region, combined linkage disequilibrium/linkage analysis (LDLA) could not increase the QTL mapping resolution (data not shown). Possibly, the marker density in the QTL region needs to be increased before the QTL can be positioned with significantly more accuracy. At present, additional markers in the QTL region are not available.

Based on four-marker haplotypes found in QTL-heterozygous parents, putative genotypes at the underlying polymorphism were deduced in parents that had been found negative in the test for QTL. As expected, a highly significant surplus of homozygous genotypes was found, although there were 13 discrepancies in the form of parents that were negative in the test for QTL while being subsequently deduced to have the heterozygous genotype. Of these 13 parents, four carried copies of the three haplotypes alleles that were ambiguous in terms of being linked to $Q$ or $q$. These four, therefore, were more likely to be true homozygotes representing ambiguous haplotypes. For the remaining nine parents, no apparent reason for their discrepancy could be found.

The QTL for IPN-resistance has now been detected in two different populations, Norwegian and Scottish, with high heterozygosity observed in both. This raises the interesting question of how an allele with a highly negative effect on a trait can be retained in populations at such large fre- quencies (especially since the mode of inheritance appears to be purely additive). One possible explanation is that the low-resistance allele has a positive effect on another trait under natural or artificial selection in these populations. This hypothesis, however, is opposed by an almost complete lack of observed negative genetic correlations between IPN-resistance and other traits recorded by Aqua Gen and other breeding companies. More specifically, among 14 recorded traits IPN resistance was found to be negatively and significantly correlated with only one trait. The trait in question was not among those that have been under strong selection, and the (genetic) correlation was also not strong $(\mathrm{r}=0.17)$ (Aqua Gen Ltd., unpublished data). It is quite possible, therefore, that this QTL is neutral under wild conditions, but non-neutral within an aquaculture setting. Disease pressures are highly different within these two settings, and IPN-resistance has in Norway been subject to artificial selection for at most two salmon generations, with only family selection being employed and IPN being only one of several traits in the breeding goals; probably not strong enough selection for large changes in allele frequency to occur.

Given that a single QTL explains such a large proportion of the genetic variance for this trait, it is reasonable to assume that the high-resistance allele will move towards fixation relatively rapidly even without MAS, although MAS can accelerate the process significantly, or permit more focus to be put on other traits without compromising the genetic gain for IPN resistance. Some researchers might argue that loss of phenotype-affecting genetic variation should be avoided, even if the allele going towards extinction has not been found to have positive effects on other traits. In such a case, the marker tools described in this paper could be used to deliberately retain the lowresistance allele at low frequency in the population.

The QTL presented here has been in use in the Aqua Gen breeding programme since 2007, when within-family MAS was done on 30 half-sib groups in order to select the most IPN-resistant fish as parents of the elite fish (i.e. the fish used for egg production). At the time, MAS could only be done within family, and only within offspring of parents that had already been screened for segregation of the QTL. Presently, MAS could be done on the bulk of the population using linkage information, since QTL genotypes and marker-QTL linkage phases were established for $72 \%$ of the parents of the current generation (a number that can be increased by adding genotypes). There is also a potential for MAS based on linkage disequilibrium, since haplotypes were found that were associated with the underlying gene at the population level. However, the markers contributing to the haplotype cover a $10 \mathrm{cM}$ interval, and are not likely to be stable over generations. Thus, there is a need to further characterise the QTL in order to 
obtain the most efficient tool for MAS, and to uncover the gene that is underlying this trait. Recent development in genomics, including an Atlantic salmon SNP-chip containing 16,000 SNPs developed at the Centre of Integrative Genetics (CIGENE; Ås, Norway), could prove highly useful in this respect. Also, samples of Aqua Gen broodstock dating as far back as the early 1980 s will be investigated in order to test the hypothesis of the high-resistance allele having increased in frequency due to aquaculture breeding.

\section{Conclusion}

In conclusion, the study described here led to the confirmation and fine-mapping of a major QTL for resistance against the viral disease IPN in Atlantic salmon. Alleles at haplotypes, constructed from microsatellite markers located in the QTL region, were found to be associated with resistance at the population level, providing a tool for MAS at the population level. To our knowledge, this is the first tool for LD-based tool for MAS developed in any aquaculture species. Further studies are now progressing, aiming at the identification of the underlying polymorphism.

\section{Methods \\ Genetic material, challenge tests}

The challenge tested fish were from the 2005 year class of the breeding company Aqua Gen AS. The parents of this year class came from four different, semi-closed, Aqua Gen populations originating from different Norwegian rivers in the early 1970s [7]. Two challenge tests were performed; one at the fry stage (four days past first feeding, first feeding occurring approximately four months past fertilisation and two months past hatching) and the other at the post-smolt stage (eight months past first feeding). In the fry-stage test, the standard test performed by the breeding company, all families from the year class were tested. In the post-smolt test, 10 selected full-sib families were tested. The selection of full-sib families for the postsmolt test was based upon family survival (most families selected being average, with one high-ranking and one low-ranking family also represented). Both challenge tests were performed at the VESO Vikan Research Station in (Namsos, Norway).

For the fry test, full-sib family groups of Atlantic salmon fry were transported from Kyrksæterøra (Norway) to VESO Vikan at Namsos (Norway). The fish were first-fed one day after arrival at Vikan, and acclimatised for four more days before challenge. The families were challenged in individual 10 liter tanks (84-126 individuals per tank). The challenge test was initiated four days past first feeding and terminated at $38.9 \%$ overall mortality. The fish were challenged with IPN virus (isolate V-1244) using bath challenge $\left(3 \mathrm{ml}\right.$ of $1.35 \times 10^{8} \mathrm{TCID} / \mathrm{ml}$ added to each tank). Dead or moribund fish from 50 randomly sampled tanks were tested for IPN infection by employing an agglutination test on ascites fluid [33] or by histopathology. All diagnostic tests for IPN were positive. The fish were fed twice a day during the challenge test. From each tank, the first 10 to die and 10 random chosen survivors were sampled and stored on $96 \%$ ethanol. DNA was extracted using the DNeasy 96 kit from QIAGEN (Venlo, the Netherlands).

The post-smolt test was performed on fish that were siblings (same spawning) of the fish from the fry test. In order to induce smoltification in fish destined for the post-smolt test, the fish were exposed to $24 \mathrm{~h}$ daylight for the last 6 weeks prior to challenge. Sea-water-ready, PITtagged, Atlantic salmon smolts (8506 individuals, average weight $71 \mathrm{~g}$, eight months past first feeding) were transported from Kyrksæterøra (Norway) to Namsos, (Norway) and distributed into 2 tanks (3 $\mathrm{m}$ diameter). Following acclimatisation for 6 days in fresh water and 3 days in salt water, 950 Atlantic salmon (average weight 58 g) infected with IPN (serotype V-1244) by intraperitoneal injection $\left(0.5 \mathrm{ml}\right.$ per animal, $6 \times 10^{7} \mathrm{TCID}_{50}$ per $\left.\mathrm{ml}\right)$ were added to each tank. Dead fish were sampled and registered on a daily basis. The test was terminated 25 days post challenge. During challenge, the fish were fed $a d$ lib. Ninety randomly sampled test fish were tested for IPNinfection using ELISA [34], and for secondary bacterial infection using bacteriological tests. All of these 90 fish were found to be IPN-positive, and negative for bacterial pathogens. Individual fish were weighed after challenge. Liver samples from challenge-tested animals were kept at $-20^{\circ} \mathrm{C}$. DNA was extracted using the DNeasy 96 kit from QIAGEN (Venlo, the Netherlands).

\section{Genome scan}

A genome scan for IPN-resistance QTL was performed on the 10 post-smolt challenged families. Within each fullsib family, only the 92 first to die and 92 randomly chosen survivors, out of 1000 challenge-tested individuals, were genotyped (selective genotyping; [35]). For the genome scan, 136 microsatellite loci were genotyped (see Additional file 1 for locus names, primer sequences, accession numbers, map positions, and multiplexes). Most of these microsatellites were selected from the 2006 version of the SALMAP female linkage map (present version: $[36,37])$, and they were selected collectively for optimal genome coverage. In addition, a number of tetra-nucleotide microsatellites that were not on the SALMAP were included in the set. Since the published map [31] contains SNP markers that were not used in the present study, map positions of markers used in the present study do not always correspond to the positions published earlier [31]. Additional file 1 contains map positions according to the 
present study and to the published map [31]. Female map distances were used.

The genome scan data were checked for non-Mendelian segregation and double recombinants (indicative of genotyping errors) as laid out in Moen et al. 2008 [31]. The data were tested for QTL using the method of Haley and Knott [38], as implemented in the half-sib module of the program package QTL Express [39]. The Haley and Knott method tests parents individually for segregation of QTL alleles, and combines these independent tests into an overall test statistic. Survival was coded as a binary trait (affected/resistant) rather than as days-in-test, since the variation in days-in-test within the two binary classes was very small compared to the variation between the classes. It has been shown that a binary trait can be analysed using QTL mapping methods intended for quantitative traits, as long as the trait is a threshold trait with an underlying normal distribution [40-42]. To accommodate joint analysis of male and female mapping parents, the data set was duplicated prior to analysis, with the designation of parents as sire or dams inverted in the duplicate. Chromosome-wide significance levels were determined by withinchromosome permutation testing with 10,000 iterations [43]. A QTL was found to be genome-wide (i.e. experiment-wide) significant if the chromosome-wide significance level was smaller than $0.05^{*} \mathrm{~L} / 1436$, where $\mathrm{L} \mathrm{cM}$ is the genetic length of the linkage group (i.e. chromosome) and $1436 \mathrm{cM}$ is the genetic length of the whole genome. QTL that were chromosome-wide but not genome-wide significant were regarded as 'suggestive' QTL. Confidence intervals for QTL position were determined by bootstrap analysis with $\geq 1000$ iterations [44]. The proportion of phenotypic variance explained by the QTL was calculated as $4^{*}\left(1-\mathrm{MSE}_{\text {full }} / \mathrm{MSE}_{\text {reduced }}\right)$ where $\mathrm{MSE}_{\text {full }}$ is the mean squared error of the full model, accommodating one QTL effect for each informative mapping parent, while $\mathrm{MSE}_{\mathrm{re}}$ duced is the corresponding mean squared error of the reduced model omitting QTL effects [38]. Selective genotyping was corrected for as proposed by Darvasi and Soller [35]. More specifically, the correction factor $\gamma_{p}$ was calculated to be 3.24 for the smolt material and 3.67 for the fry material; the uncorrected variance proportions were divided by this factor to obtain the corrected variance proportions. For the calculations, it was assumed that the $9.2 \%$ least and $9.2 \%$ most resistance animals were genotyped for post-smolts $(7.7 \%$ upper and $7.7 \%$ lower for fry). Since in reality, the set of "most resistant animals" were drawn randomly from the $\sim 25 \%$ (average) of animals that survived within family, the factor is likely to be an underestimate in the post-smolt data set. The proportion of genetic variance explained by the QTL was found by dividing the proportion of phenotypic variance with the heritability, estimated to be 0.35 based on the 2005 fry challenge test (Aqua Gen AS, unpublished data). A her- itability estimate for IPN-resistance in post-smolts was not available.

\section{Test for QTL on individual parents}

To identify QTL-heterozygotes, individual mapping parents were tested for segregation of a major QTL at individual markers or haplotypes using a two-way chi-square test. A parent was defined as QTL heterozygous if this test was significant at $\mathrm{P}<0.05$.

\section{Linkage-based fine mapping of QTL using post-smolt (genome scan) material and fry material}

To map a QTL on linkage group 21 with more precision, 18 additional microsatellites mapping to this linkage group were identified using the SALMAP map (Roy Danzmann, pers. comm., [36]) The identities, primer sequences, multiplexes and annealing temperatures of the microsatellites can be found in Additional file 1. Microsatellite genotyping and QTL analysis were performed as for the genome scan.

\section{Construction of haplotypes, determination of linkage between haplotypes and QTL-alleles, and estimation of inter-marker linkage disequilibrium}

Haplotypes were constructed from sets of microsatellites defining the region of a major QTL. This was done using all fry data and a custom-made computer program described in [31]. Briefly, the program performed these steps (within every mapping parent): 1) Start at the first informative marker from one end of the linkage group; 2 ) find the linkage phase between that marker and the next informative marker, minimising the number of recombination events in the offspring; 3 ) proceed in this manner to find the linkage phase between all informative marker, and thus to build the two haplotypes; and 4) for monomorphic markers, insert the same allele in both haplotypes. To link individual haplotype alleles, or single marker alleles, to alleles at the underlying polymorphism, QTL-heterozygous parents of the challenge-tested fry families (those with evidence of being QTL-heterozygous at P $<0.05)$ were identified, whereupon their haplotype/ marker alleles were designated as being linked to the highresistance $(Q)$ or low-resistance allele $(q)$ allele based on the fry data. The association between haplotype/marker alleles and the $Q / q$ status of the chromosomes on which the alleles resided were tested for using the GLM procedure of SAS (SAS Institute Inc., Cary, NC), with allele as the only (fixed) effect in the model. The effect of deduced allele at the underlying polymorphism on survival rate of offspring groups was tested for using the GLM procedure of SAS, with fixed effects of full-sib group, number of copies of the high-resistance allele $(0,1$, or 2 within every parent), and heterozygous state ( 0 if the parent was heterozygous for the underlying polymorphism, 1 otherwise). 
The LD measure was $\mathrm{r}^{2}$, calculated as the square of Cramer's V [45] using the function haploxt of the program GOLD [46]. The sampling effect was corrected for by subtracting $1 /$ (number of haplotypes) from $\mathrm{r}^{2}[47]$. A $6^{\text {th }}$ degree polynomial was fitted to the data. We did not attempt to fit a parametric function, such as the one of Sved [48], to the data, since the population is a recently admixed population [31].

\section{Authors' contributions}

TM initiated the project, was project leader, co-supervised the laboratory work, processed raw data, did all data analysis except LDLA (AKS) and analysis of post-smolt data (contributions from SK), and wrote a draft of the manuscript with contributions from the other authors. $\mathrm{MB}$ cosupervised the laboratory work, developed microsatellite protocols and multiplexes, and processed raw data. AKS did LDLA. SK collected and processed phenotypic data, and coordinated the processing of genetic material. All authors read and approved of the manuscript.

\section{Additional material}

\section{Additional file 1}

Microsatellite markers used. Names, genome positions, PCR conditions, primer sequences, references, and accession numbers of microsatellite markers used in the study.

Click here for file

[http://www.biomedcentral.com/content/supplementary/14712164-10-368-S1.xls]

\section{Additional file 2}

Information content and statistics from genome scan. Information content and statistics from the genome scan.

Click here for file

[http://www.biomedcentral.com/content/supplementary/14712164-10-368-S2.xls]

\section{Additional file 3}

Marker/haplotype alleles among QTL heterozygous parents classified according to genotype at the underlying polymorphism. Marker and haplotype alleles were linked to alleles at the underlying polymorphism (Q $=$ high-resistance allele, $\mathrm{q}=$ low-resistance allele) using 72 parents identified as QTL-heterozygous $(P<0.05) .{ }^{*}$ Marker/haplotype alleles significantly associated with QTL alleles (binomial test, $P<0.05$ ).

Click here for file

[http://www.biomedcentral.com/content/supplementary/14712164-10-368-S3.ppt]

\section{Acknowledgements}

We wish to thank Hege Munck (Nofima) and Katrine Hånes (Nofima) for doing the bulk of genotyping work; Elin Bergseth (BioBank AS, Hamar) for processing and weighing of tissue samples; Arne Storset (Aqua Gen) for taking part in the initiation of the project, for useful advice throughout the project period, and for commenting on the manuscript; Ben Hayes (DPI, Melbourne) for taking part in the initiation of the project; Roy Danzmann (University of Guelph, Canada) for providing details on microsatellite mark- ers; Olai Einen (Nofima) for taking part in the initiation of the project; Olaf Skjærvik (VESO AS) for coordinating challenge tests and for commenting on the manuscript; Theo Meuwissen for commenting on the manuscript; Sigbjørn Lien and Stig Omholt for providing laboratory facilities; and two anonymous reviewers for providing valuable comments on earlier versions of the manuscript. This project was funded by HAVBRUK programme of the Research Council of Norway (project 169522/S40) and Aqua Gen AS.

\section{References}

I. Wedemeyer G: Physiology of fish in intensive culture systems London: Chapman \& Hall; 1996.

2. Duarte CM, Marbá N, Holmer M: Rapid Domestication of Marine Species. Science 2007, 316:383-383.

3. Ariel E, Olesen NJ: Finfish in aquaculture and their diseases - a retrospective view in the European Community. Bulletin of the European Association of Fish Pathologists 2002, 22:72-85.

4. Murray AG, Busby CD, DW B: Infectious pancreatic necrosis on Scottish salmon farms 1996-200I. Emerging Infectious Diseases 2003, 9:2-31.

5. Brun E: Epidemiology. In IPN in salmonids: A review Edited by: Skjelstad B. Oslo: The Fisheriers and Aquaculture Industries Research Fund (FHF); 2003.

6. Mikalsen AB, Torgersen J, Alestrom P, Hellemann AL, Koppang EO, Rimstad E: Protection of Atlantic salmon Salmo salar against infectious pancreatic necrosis after DNA vaccination. Diseases of aquatic organisms 2004, 60: II-20.

7. Gjedrem T, Gjøen HM, Gjerde B: Genetic origin of Norwegian farmed Atlantic salmon. Aquaculture 199I, 98:4I-50.

8. AquaBreeding survey on the Breeding Practices in the European aquaculture industry [http://aquabreeding.eu/Portals/2/ AquaBreeding\%20Survey\%20-\%20Updated\%20version\%202009.pdf]

9. Ozaki A, Khoo SK, Yoshiura Y, Ototake M, Sakamoto T, Dijkstra JM, Okamoto N: Identification of Additional Quantitative Trait Loci (QTL) Responsible for Susceptibility to Infectious Pancreatic Necrosis Virus in Rainbow Trout. Gyobyo kenkyu 2007, 42: $131-140$.

10. Ozaki A, Sakamoto T, Khoo SK, Nakamura K, Coimbra MRM, Akutsu T, Okamoto N: Quantitative trait loci (QTLs) associated with resistance/susceptibility to infectious pancreatic necrosis virus (IPNV) in rainbow trout (Oncorhynchus mykiss). Mol Genet Genomics 200I, 265:23-3I.

11. Palti Y, Nichols KN, Waller KI, Parsons JE, Thorgaard GH: Association between DNA polymorphisms tightly linked to MHC class II genes and IHN virus resistance in backcrosses of rainbow and cutthroat trout. Aquaculture 200I, 194:283-289.

12. Palti $\mathrm{Y}$, Parsons JE, Thorgaard GH: Identification of candidate DNA markers associated with IHN virus resistance in backcrosses of rainbow (Oncorhynchus mykiss) and cutthroat trout (O clarki). Aquaculture 1999, I73:81-94.

13. Langefors $\AA$, Lohm J, Grahn M, Andersen $\varnothing$, von Schantz T: Association between major histocompatibility complex class IIB alleles and resistance to Aeromonas salmonicida in Atlantic salmon. Proc $R$ Soc Lond B 200I, 268:479-485.

14. Miller KM, Winton JR, Schulze AD, Purcell MK, Ming TJ: Major histocompatibility complex loci are associated with susceptibility of Atlantic salmon to infectious hematopoietic necrosis virus. Environmental Biology of Fishes 2004, 69:307-316.

15. Khoo SK, Ozaki A, Nakamura F, Arakawa T, Ishimoto S: Identification of a novel chromosomal region associated with IHNV resistance in rainbow trout. Fish Pathology 2004, 39:95-102.

16. Moen T, Fjalestad KT, Munck H, Gomez-Raya L: A Multistage Testing Strategy for Detection of Quantitative Trait Loci Affecting Disease Resistance in Atlantic Salmon. Genetics 2004, 167:85I-858.

17. Moen T, Sonesson AK, Hayes B, Lien S, Munck H, Meuwissen THE: Mapping of a quantitative trait locus for resistance against infectious salmon anaemia in Atlantic salmon (Salmo Salar): comparing survival analysis with analysis on affected/resistant data. BMC Genetics 2007, 8:53.

18. Nichols KM, Bartholomew J, Thorgaard GH: Mapping multiple genetic loci associated with Ceratomyxia shasta resistance in Ochorynchus mykiss. Dis Aquat Org 2003, 56: I45-154. 
19. Zimmerman AM, Evenhuis JP, Thorgaard GH, Ristow SS: A single major chromosomal region controls natural killer cell-like activity in rainbow trout. Immunogenetics 2004, 55:825-835.

20. Barroso R, Wheeler PA, Lapatra S, Drew RE, Thorgaard GH: QTL for IHNV resistance and growth identified in a rainbow (Oncorhynchus mykiss) $X$ Yellowstone cutthroat (Oncorhynchus clarki bouvieri) trout cross. Aquaculture 2008, 277:156-163.

21. Gilbey J, Verspoor E, Mo TA, Sterud E, Olstad K, Hytterød S, Jones C, Noble L: Identification of genetic markers associated with Gyrodactylus salaris resistance in Atlantic salmon Salmo salar. Dis Aquat Organ 2006, 71 : I 19-129.

22. Rodriguez MF, Lapatra S, Williams S, Famula T, May B: Genetic markers associated with resistance to infectious hematopoietic necrosis in rainbow and steelhead trout (Oncorhynchus mykiss) backcrosses. Aquaculture 2005, 241:93-II5.

23. Grimholt U, Larsen S, Nordmo R, Midtlyng P, Kjøglum S, Storset A, Sæbø S, Stet RJM: MHC polymorphism and disease resistance in Atlantic salmon (Salmo salar); facing pathogens with single expressed major histocompatibility class I and class II loci. Immunogenetics 2003, 55:210-219.

24. Kjøglum S, Larsen S, Bakke HG, Grimholt U: How specific MHC class I and class II combinations affect disease resistance against infectious salmon anaemia in Atlantic salmon (Salmo salar). Fish \& Shellfish Immunology 2006, 21 :43 I-44I.

25. Houston RD, Haley CS, Hamilton A, Guy DR, Tinch AE, Taggard JB, McAndrew BJ, Bishop SC: Major quantitative trait loci affect resistance to infectious pancreatic necrosis in Atlantic salmon (Salmo salar). Genetics 2008, I78: I 109- I II5.

26. Beavis WD: The power and deceit of QTL experiments: lessons from comparative QTL studies. 49th Annual Corn and Sorghum Industry Research Conference ASTA, Washington DC 1994:250-266.

27. Georges M, Nielsen D, Mackinnon M, Mishra A, Okimoto R, Pasquino AT, Sargeant LS, Sorensen A, Steele MR, Zhao X, et al.: Mapping quantitative trait loci controlling milk production in dairy cattle by exploiting progeny testing. Genetics 1995, 139:907-920.

28. Xu S: Theoretical basis of the Beavis effect. Genetics 2003, 165:2259-2268.

29. Bootland LM, Dobos P, Stevenson RMW: Fry age and size effects on immersion immunization of brook trout, Salvelinus fontinalis Mitchell, against infectious pancreatic necrosis virus. Journal of Fish Diseases 1990, 13: I 13-125.

30. Tatner MF: Natural changes in the immune system of fish. In The Fish Immune System: Organism, Pathogen, and Environment Edited by: Iwama G, Nakanishi T. San Diego, CA: Academic Press, Inc; 1996:255-287.

31. Moen T, Hayes B, Baranski M, Berg PR, Kjøglum S, Koop BF, Davidson WS, Omholt SW, Lien S: A linkage map of the Atlantic salmon (Salmo salar) based on EST-derived SNP markers. BMC Genomics 2008, 9:223.

32. Zapata A, Diez B, Cejalvo T, Gutierrez-de Frýas C, Corte A: Ontogeny of the immune system of fish. Fish \& Shellfish Immunology 2006, I 2: I26-136.

33. Taksdal T, Thorud K: Evaluation of a rapid coagglutination (COA) test for the detection of infectious pancreatic necrosis virus (IPNV) in tissue samples of Atlantic salmon, Salmo salar L. Journal of Fish Diseases 1999, 22: I 17-124.

34. Ramstad A, Romstad AB, Knappskog DH, Midtlyng PJ: Field validation of experimental challenge models for IPN vaccines. Journal of Fish Diseases 2007, 30:723-73I.

35. Darvasi A, Soller M: Selective genotyping for determination of linkage between a marker locus and a quantitative trait locus. Theoretical and Applied Genetics 1992, 85:353-359.

36. ASalBase [http://www.asalbase.org]

37. Danzmann RG, Davidson EA, Ferguson MM, Gharbi K, Hoyheim B, Koop BF, Lien S, Lubieniecki KP, Moghadam HK, Park J, et al.: Distribution of ancestral proto-Actinopterygian chromosome arms within the genomes of $4 R$-derivative salmonid fishes (Rainbow trout and Atlantic salmon). BMC Genomics 2008, 9:557.

38. Knott SA, Elsen JM, Haley CS: Methods for multiple-marker mapping of quantitative trait loci in half-sib populations. Theoretical and applied genetics 1996, 93:71-80.
39. Seaton G, Haley CS, Knott SA, Kearsey M, Visscher PM: QTL Express: mapping quantitative trait loci in simple and complex pedigrees. Bioinformatics 2002, 18:339-340.

40. Visscher PM, Haley CS, Knott SA: Mapping QTLs for binary traits in backcross and F2 populations. Genetical Research 1996, 68:55-63.

4I. Kadarmideen HM, Dekkers JCM: Generalized marker regression and interval QTL mapping methods for binary traits in halfsib family designs. J Anim Breed Genet 200I, I I 8:297-309.

42. Zhang C, de Koning D-J, Hernández-Sánchez J, Haley CS, Williams JL, Wiener P: Mapping of multiple quantitative trait loci affecting bovine spongiform encephalopathy. Genetics 2004, 167: $1863-1872$

43. Churchill GA, Doerge RW: Empirical threshold values for quantitative mapping. Genetics 1994, I38:963-97I.

44. Visscher PM, Thompson R, Haley RS: Confidence intervals in QTL mapping by bootstrapping. Genetics 1996, I43: I0 I3-1020.

45. Cramer H: Mathematical Models of Statistics Princeton, New Jersey, USA: Princeton University Press; 1946.

46. Abecasis GR, Cookson WO: GOLD-graphical overview of linkage disequilibrium. Bioinformatics 2000, 16:182-183.

47. Hill WG: Estimation of effective population size from data on linkage disequilibrium. Genetical Research |98I, 38:209-216.

48. Sved JA: Linkage disequilibrium and homozygosity of chromosome segments in finite populations. Theoretical Population Biology |97|, 2:|24-|4|.
Publish with Biomed Central and every scientist can read your work free of charge

"BioMed Central will be the most significant development for disseminating the results of biomedical research in our lifetime. "

Sir Paul Nurse, Cancer Research UK

Your research papers will be:

- available free of charge to the entire biomedical community

- peer reviewed and published immediately upon acceptance

- cited in PubMed and archived on PubMed Central

- yours - you keep the copyright

Submit your manuscript here:

http://www.biomedcentral.com/info/publishing_adv.asp
BioMedcentral 